\title{
H-dibaryon matter in the Skyrme model on a hypersphere
}

\author{
T. Sakai* and H. Suganuma \\ RCNP, Osaka University, Ibaraki, Osaka 567-0047, Japan
}

November 10, 2018

\begin{abstract}
We study the properties of H-dibaryon matter through the $\mathrm{SO}(3)$ Skyrmion solution on a three-dimensional hypersphere $S^{3}$. As the density increases, the swelling of H-dibaryon is found. Above a critical density, the system becomes uniform in terms of the baryon density. In this uniform phase, the critical order parameter is largely reduced, which can be interpreted as the chiral symmetry restoration. From the comparison with the $\mathrm{SO}(2) \times \mathrm{SO}(2)$ solution, the $\mathrm{SO}(3)$ soliton is found to be the true ground state for high density system with $R \leq 0.64 \mathrm{fm}$ in the $B=2$ sector with $N_{\mathrm{f}}=3$.
\end{abstract}

\section{Introduction}

Recently exotic hadron states have been studied elaborately. Among them, an H-dibaryon has been an object of many works [1] as a hopeful candidate of $q^{6}$ state since the prediction by Jaffe 2] in the MIT bag model. Lots of theoretical studies have been made on the problem of the H-dibaryon, and have predicted the H-dibaryon mass below the $\Lambda \Lambda$ threshold [1], 3]. The Skyrme model was first applied to the H-dibaryon by Balachandran et al. proposing the $B=2 \mathrm{SO}(3)$ soliton configuration, which has the symmetric configuration in terms of the flavor SU(3) [⿴囗十 , 5]. They obtained the H-dibaryon mass lighter than two $\Lambda$ 's $\left(M_{\mathrm{H}} \sim 1.92 M_{\mathrm{B}}\right)$ in the $\mathrm{SU}(3)$ flavor symmetric limit.

Experimentally, several candidate events have been reported [6, 7], but any conclusive result for its existence is not obtained yet. The binding energy of two $\Lambda$ 's in a double hypernucleus places a restriction on the H-mass. A double hypernucleus event found at KEK 8 implies that the lower limit of the H-dibaryon mass is a few tens of $\mathrm{MeV}$ below the $\Lambda \Lambda$ threshold.

The possibility of the existence of H-dibaryon matter in a high-density neutron star is pointed out by Tamagaki et al. [9]. In their argument, the compactness of the H-dibaryon radius is an important assumption on the existence of H-dibaryon matter, although the size of H-dibaryon may vary in the dense system as the nucleon swelling phenomenon in nuclear matter[10]. If so, nuclear matter may decay into H-matter at a critical density $\rho_{\mathrm{c}}=6 \sim 9 \rho_{0}$ $\left(\rho_{0}=0.17 \mathrm{fm}^{-3}\right)$ before the phase transition into quark-gluon plasma.

\footnotetext{
*E-mail: tsakai@rcnp.osaka-u.ac.jp
} 
The properties of nuclear matter at high densities have been investigated in many ways. Castillejo et al. studied Skyrmion matter by placing SU(2) hedgehog Skyrmions on a lattice and show that the deconfinement phase transition and the chiral symmetry restoration occur above a critical density [1]. Manton and Ruback obtained the similar results by a simple and mathematically sophisticated approach. Instead of placing many Skyrmions in the flat space $\boldsymbol{R}^{3}$, Skyrmion matter is approximated by one Skyrmion on an $S^{3}$ hypersphere with radius $R$. They showed that beyond a critical density the lowest energy solution is given by the "identity map" and the baryon density distributes uniformly on the hypersphere[12]. In this phase, the spacially averaged value of the chiral order parameter becomes zero, which may imply the chiral symmetry restoration at high densities. This phase transition was investigated also in the case of two Skyrmions on $S^{3}\left[13\right.$. The result is qualitatively the same as one Skyrmion case on $S^{3}$, and they concluded that these behaviors are a general tendency in the Skyrme model.

In this paper, we investigate the feature of H-dibaryon matter by using the SO(3) Skyrmion with baryon number 2 located on $S^{3}$ hypersphere. We study how the QCD phase transition occurs in this situation. We study also how the size of the H-dibaryon varies in high-density H-matter. For simplicity on the mathematical and physical insight, we deal with the chiral limit in this paper.

\section{Formalism}

The Skyrme Lagrangian in the chiral limit [4, 15] is given by

$$
\mathcal{L}=-\frac{f_{\pi}^{2}}{2} \operatorname{Tr}\left(\partial_{\mu} U^{\dagger} \partial^{\mu} U\right)+\frac{1}{32 e^{2}} \operatorname{Tr}\left[\partial_{\mu} U U^{\dagger}, \partial_{\nu} U U^{\dagger}\right]^{2}+\mathcal{L}_{\mathrm{WZ}}
$$

where $f_{\pi}$ and $e$ denote the pion decay constant and the Skyrme parameter, respectively. Here, $U(\boldsymbol{x}) \equiv e^{i \frac{\lambda^{a}}{2} \pi^{a}(\boldsymbol{x}) / f_{\pi}} \in \mathrm{SU}(3)_{\mathrm{f}}$ is described by the Nambu-Goldstone mode, $\pi^{a}(\boldsymbol{x})(a=1, \cdots, 8)$, and $\mathcal{L}_{\mathrm{WZ}}$ denotes the Wess-Zumino term. The ansatz for the chiral field of $\mathrm{SO}(3)$ soliton on $S^{3}$ is represented with two profile functions, $\chi(\mu)$ and $\psi(\mu)$, as $[4$, 5]

$$
U=\exp \left[i\left\{\boldsymbol{\Lambda} \cdot \hat{\boldsymbol{r}} \chi(\mu)-\left(\frac{3}{2}(\boldsymbol{\Lambda} \cdot \hat{\boldsymbol{r}})^{2}-1\right) \psi(\mu)\right\}\right]
$$

Here, $\boldsymbol{\Lambda}=\left(\Lambda_{1}, \Lambda_{2}, \Lambda_{3}\right)=\left(\lambda_{7},-\lambda_{5}, \lambda_{2}\right)$ is the generators of the $\mathrm{SO}(3)$ group, where $\lambda_{a}(a=$ $1,2, \cdots, 8)$ are Gell-Mann matrices, and three polar coordinates on $S^{3}$ are $\mu$, $\theta$, and $\phi$. The baryon current density $B^{\nu}(\mu)$ and the energy of the Skyrmion $E(U)$ on $S^{3}$ with radius $R$ can be easily obtained from those on $\boldsymbol{R}^{3}[$ [ by simple replacement [14],

$$
\begin{aligned}
& r \rightarrow R \sin \mu, \\
& \mathrm{d} r \rightarrow R \mathrm{~d} \mu, \\
& \mathrm{d} V=4 \pi r^{2} \mathrm{~d} r \rightarrow \mathrm{d} V=4 \pi R^{3} \sin ^{2} \mu \mathrm{d} \mu .
\end{aligned}
$$

The winding number, which is identified with the baryon number, is

$$
B=4 \pi R^{3} \int_{0}^{\pi} \sin ^{2} \mu \mathrm{d} \mu B^{0}(\mu)
$$


where $B^{0}(\mu)$ is the baryon number density,

$$
B^{0}(\mu)=\frac{\epsilon^{0 \alpha \beta \gamma}}{24 \pi^{2}} \operatorname{Tr}\left[L_{\alpha} L_{\beta} L_{\gamma}\right]=-\frac{1}{2 \pi^{2} R^{3} \sin ^{2} \mu}\left[\frac{\mathrm{d} \chi}{\mathrm{d} \mu}\left(1-\cos \chi \cos \frac{3}{2} \psi\right)+\frac{3}{2} \frac{\mathrm{d} \psi}{\mathrm{d} \mu} \sin \chi \sin \frac{3}{2} \psi\right],
$$

with $L_{\alpha}=U^{\dagger} \partial_{\alpha} U$. For the $B=2$ Skyrmion on $S^{3}$, the profile functions satisfy the boundary conditions 四,

$$
\chi(0)=\pi, \quad \psi(0)=\frac{2}{3} \pi, \quad \chi(\pi)=0, \quad \psi(\pi)=0 .
$$

The static energy of the $\mathrm{SO}(3)$ Skyrmion on $S^{3}$ is written as

$$
\begin{aligned}
E(U) & =\int \mathrm{d} V\left[\frac{f_{\pi}^{2}}{2} \operatorname{Tr}\left(\partial_{\mu} U^{\dagger} \partial^{\mu} U\right)-\frac{1}{32 e^{2}} \operatorname{Tr}\left[\partial_{\mu} U U^{\dagger}, \partial_{\nu} U U^{\dagger}\right]^{2}\right] \\
& \equiv f_{\pi}^{2} R I_{1}+\frac{1}{e^{2} R} I_{2}
\end{aligned}
$$

where $I_{1}$ and $I_{2}$ are dimensionless integrals,

$$
\begin{aligned}
& I_{1}=4 \pi \int_{0}^{\pi} \mathrm{d} \mu \sin ^{2} \mu\left[\left(\frac{\mathrm{d} \chi}{\mathrm{d} \mu}\right)^{2}+\frac{3}{4}\left(\frac{\mathrm{d} \psi}{\mathrm{d} \mu}\right)^{2}+\frac{4}{\sin ^{2} \mu}\left\{1-\cos \chi \cos \frac{3}{2} \psi\right\}\right] \\
& I_{2}=2 \pi \int_{0}^{\pi} \mathrm{d} \mu {\left[\frac{1}{\sin ^{2} \mu}\left\{3 \sin ^{2} \chi \sin ^{2} \frac{3}{2} \psi+\left(1-\cos \chi \cos \frac{3}{2} \psi\right)^{2}\right\}\right.} \\
&+\left.\left(1-\cos \chi \cos \frac{3}{2} \psi\right)\left\{\left(\frac{\mathrm{d} \chi}{\mathrm{d} \mu}\right)^{2}+\frac{9}{4}\left(\frac{\mathrm{d} \psi}{\mathrm{d} \mu}\right)^{2}\right\}+3 \sin \chi \sin \frac{3}{2} \psi \frac{\mathrm{d} \chi}{\mathrm{d} \mu} \frac{\mathrm{d} \psi}{\mathrm{d} \mu}\right] .
\end{aligned}
$$

The Euler-Lagrange equations for $\chi$ and $\psi$ are obtained by extremizing the static energy with respect to them.

\section{$3 \quad$ Numerical results and concluding remarks}

The profile functions are obtained numerically by extremizing the energy functional $E(U)$ under the boundary conditions (6). We show in Fig.1 the profile functions of the $B=2 \mathrm{SO}(3)$ Skyrmion on $S^{3}$ in typical two cases using the parameters in ref. [4]: $f_{\pi}=67 \mathrm{MeV}$ and $e=4.47$. (In Refs. 12, 13], the energy scale is rescaled by $f_{\pi} /(\sqrt{2} e)=10.6 \mathrm{MeV}$, and the length scale by $1 /\left(\sqrt{2} e f_{\pi}\right)=0.466 \mathrm{fm}$ to reduce variable dimensionless.) There are always two physically

equivalent solutions: one is localized around the north pole, and another is localized around the south pole when $R$ is large. The two solutions, $\left(\chi_{1}, \psi_{1}\right)$ and $\left(\chi_{2}, \psi_{2}\right)$, have the same energy, and have the relation,

$$
\begin{aligned}
\chi_{2}(\mu) & =\pi-\chi_{1}(\pi-\mu), \\
\psi_{2}(\mu) & =\frac{2}{3} \pi-\psi_{1}(\pi-\mu) .
\end{aligned}
$$

At low densities, the Skyrmion localizes at either pole. In Fig.1(a), the profile functions for $R=2 \mathrm{fm}$ are shown, where the Skyrmion is localized around the south pole. The behavior 
of the profile functions is similar to that for the Skyrmion on the flat space $\boldsymbol{R}^{3}[5]$. On the other hand, as shown in Fig.1(b), the profile functions spread all over the hypersphere at high densities.

Fig.2 shows the energy of the $\mathrm{SO}(3)$ Skyrmion solution with the lowest energy as a function of the hypersphere radius $R$. At large $R$, the energy of the Skyrmion on $S^{3}$ approaches the one on $\boldsymbol{R}^{3}$. The behavior of the energy is essentially parallel with the $\mathrm{SU}(2)$ Skyrmion on $S^{3}[12]$. The character of the lowest energy solution changes around $R \sim 1 \mathrm{fm}$. The minimum energy is attained at $R \simeq 0.57 \mathrm{fm}$, and the minimum value is only $4 \%$ above the BPS saturation value $E_{\mathrm{BPS}} \equiv 6 \sqrt{2} \pi^{2}\left(f_{\pi} / e\right)|B|$ (Bogomol'ny bound). It can be shown that the BPS saturation 15 is never attained for the $\mathrm{SO}(3)$ Skyrmion on $S^{3}$ unlike the $\mathrm{SU}(2)$ Skyrmion on $S^{3}$. In the $B=1$ $\mathrm{SU}(2)$ case, the BPS saturation is achieved by the "identity map", $U(\mu, \theta, \varphi)=\cos f(\mu)+i \boldsymbol{\tau}$. $\hat{\boldsymbol{r}} \sin f(\mu)$ with $f(\mu)=\mu$ or $f(\mu)=\pi-\mu$ [12]. The $\mathrm{SU}(2)$ solution on $S^{3}$ becomes "identity map" for small $S^{3}$ as $R \leq R_{\mathrm{c}}$. On the other hand, in the $\mathrm{SO}(3)$ soliton solution, there are two kinds of profile functions, $\chi(\mu)$ and $\psi(\mu)$, and the $B=2 \mathrm{SO}(3)$ solution on $S^{3}$ tends to approach $\chi(\mu)=\frac{3}{2} \psi(\mu)=\pi-\mu$ as $R$ decreases as shown in Figs.1(a) and (b). Moreover, there is a similarity in that the profile function has linear dependence on $\mu$, so that we call the $B=2$ $\mathrm{SO}(3)$ solution with $\chi(\mu)=\frac{3}{2} \psi(\mu)=\pi-\mu$ as the "identity map" in this paper. Being different from the $\mathrm{SU}(2)$ case, the "identity map" does not satisfy the Euler-Lagrange equations so that it is slightly above the lowest energy curve at high densities.

At the low density phase, the baryon density is localized around either the north or the south pole, while at the high density phase the SO(3) Skyrmion spreads over the hypersphere, and loses its identity as a soliton. This may correspond to a phase transition from the confinement phase to the deconfinement phase. In Fig.3, we show the baryon number densities $B^{0}(\mu)$ of the solutions of several hypersphere radii around this phase transition. We can clearly see the localized-delocalized transition.

The phase transition occurs so smoothly that it is difficult to find the critical density strictly. If we put the critical radius $R_{\mathrm{c}}$ to be $0.8 \sim 1.0 \mathrm{fm}$, the corresponding critical density $\rho_{\mathrm{c}}=$ $2 /\left(2 \pi^{2} R_{\mathrm{c}}^{3}\right)$ is $0.1 \sim 0.2$ baryons $/ \mathrm{fm}^{3}$, which is the same order of magnitude as the equilibrium nuclear matter density $\rho_{0}$. This is rather smaller value than the expected critical density which is the order of several times $\rho_{0}$. Too low critical density problem appears also in $B=1$ hedgehog Skyrmion matter 11, 12, 13. The critical density $\rho_{\mathrm{c}}$ is $0.07 \sim 0.18 \mathrm{fm}^{-3}$ in these models. One of the reason is probably the lack of the kinetic energy as stated in ref.[13]. Nevertheless, it is interesting to be successful in modeling the high density phase transition in QCD qualitatively by such simple models.

The order parameter $\sigma_{0}$, which is a measure of the chiral symmetry breaking, is introduced by the spacial average 16,

$$
\sigma_{0} \equiv\left|\int_{S^{3}} \mathrm{~d} V \frac{1}{3} \operatorname{Tr} U\right|=\frac{2}{\pi}\left|\int_{0}^{\pi} \sin ^{2} \mu \mathrm{d} \mu\left(\frac{1}{3} e^{i \psi}+\frac{2}{3} \cos \chi e^{-\frac{i}{2} \psi}\right)\right| .
$$

As shown in Fig.4, the order parameter $\sigma_{0}$ becomes smaller at high densities, which is considered to be the sign of the chiral symmetry restoration. The non-zero value of the order parameter may be due to the limitation of the non-linear sigma model. 
The Skyrmion root mean square (r.m.s.) radius is given by

$$
\bar{r} \equiv \sqrt{\left\langle(R \sin \mu)^{2}\right\rangle}=\sqrt{\int_{S^{3}} \mathrm{~d} V B^{0}(\mu)(R \sin \mu)^{2} / 2}
$$

and shown in Fig.5. The contributions from the upper half $(0 \leq \mu \leq \pi / 2)$ and the lower half $(\pi / 2 \leq \mu \leq \pi)$ of the hypersphere are also shown. At low densities, $\bar{r}$ is almost determined by the contribution from the half of the hypersphere where the Skyrmion localizes, while the both contributions are almost the same at high densities. Thus the deconfinement phase transition is read also in Fig.5. At low densities, the Skyrmion size coincides with the size of the Skyrmion on $\boldsymbol{R}^{3}$. In the localized phase $\left(R \geq R_{\mathrm{c}} \simeq 0.8-1.0 \mathrm{fm}\right)$, the size $\bar{r}$ of the $\mathrm{SO}(3)$ Skyrmion becomes larger corresponding to the spread of its profile functions for small $R$ or at high densities. This enlargement implies the swelling of the H-dibaryon in high density $\mathrm{H}$-dibaryon matter. In the deconfinement phase, where the Skyrmion loses its identity as the soliton, $\bar{r}$ decreases linearly with the hypersphere radius $R$.

Finally, we compare the $\mathrm{SO}(3)$ soliton solution with the $\mathrm{SO}(2) \times \mathrm{SO}(2)$ one, which is also a candidate of the ground state of the $B=2$ Skyrmion on a small hypersphere $S^{3}$ in the two-flavor case [13]. We show in Fig.6 the total energy as a function of the hypersphere radius $R$ for the $\mathrm{SO}(3)$ and the $\mathrm{SO}(2) \times \mathrm{SO}(2)$ soliton solutions in the $B=2$ sector on $S^{3}$. The energy difference is found to be quite small between the $\mathrm{SO}(3)$ and the $\mathrm{SO}(2) \times \mathrm{SO}(2)$ solutions for the high density region as $R \lesssim 1 \mathrm{fm}$. For instance, one finds $0.90<E_{\mathrm{SO}(3)} / E_{\mathrm{SO}(2) \times \mathrm{SO}(2)}<1.03$ for $0.2 \leq R \leq 1.0 \mathrm{fm}$. One also finds $E_{\mathrm{SO}(3)} \geq 1.041 E_{\mathrm{BPS}}$ and $E_{\mathrm{SO}(2) \times \mathrm{SO}(2)} \geq 1.045 E_{\mathrm{BPS}}$. From the detailed analyses, the $\mathrm{SO}(3)$ solution is the true ground state for $R \geq 0.99 \mathrm{fm}$ (dilute case) and $R \leq 0.64 \mathrm{fm}$ (dense case), while the $\mathrm{SO}(2) \times \mathrm{SO}(2)$ solution is more stable for the intermediate density regions as $0.64 \leq R \leq 0.99 \mathrm{fm}$. Thus, the $\mathrm{SO}(3)$ soliton solution is the true ground state for the small hypersphere with $R<0.64 \mathrm{fm}$ in the $N_{\mathrm{f}}=3$ chiral limit in the mathematical sense. In the real world, however, the large symmetry breaking in the strangeness sector should enhance $E_{\mathrm{SO}(3)}$ largely, and therefore the $\mathrm{SO}(2) \times \mathrm{SO}(2)$ Skyrmion may become more stable.

We next consider the chiral order parameter $\sigma_{0}$ in eq.(11). Since the $\mathrm{SO}(2) \times \mathrm{SO}(2)$ solution takes the form as $U=\left(\begin{array}{cc}e^{i \boldsymbol{\tau} \cdot \boldsymbol{\pi}} & 0 \\ 0 & 1\end{array}\right)$ with $\int_{S^{3}} e^{i \boldsymbol{\tau} \cdot \boldsymbol{\pi}} d V=0$ 13 independent of $R, \sigma_{0}=\frac{1}{3}$. It is to be noted that the $\mathrm{SO}(2) \times \mathrm{SO}(2)$ solution on $S^{3}$ provide the "chiral symmetric phase" only in the two-flavor sector, and does not lead to the $N_{\mathrm{f}}=3$ chiral symmetric phase.

In summary, the $\mathrm{SO}(3)$ Skyrmion solution with $B=2$ has been investigated on a hypersphere $S^{3}$ to study high density H-dibaryon matter. In this system, the deconfinement transition and the chiral symmetry restoration have been found at high densities similarly to $B=1 \mathrm{SU}(2)$ Skyrmion matter. As a remarkable fact, the H-dibaryon in H-matter swells at high densities. The swelling phenomenon is considered to reflect the attractive nature of the interaction between H-dibaryons [17]. Such a possibility of the swelling of the H-dibaryon would be important for the study of H-matter in dense neutron stars, because the compactness of the H-dibaryon is a basic assumption for the existence of H-matter. For the realistic treatment of $\mathrm{H}$-dibaryon, we need to take account of the $\mathrm{SU}(3)$ flavor symmetry breaking. This will be done in the future work. 
Figure 1: The profile functions, $\chi(\mu)$ and $\psi(\mu)$, of the $B=2 \mathrm{SO}(3)$ Skyrmion on $S^{3}$ vs. the angle $\mu$ of the hypersphere for (a) $R=2.0 \mathrm{fm}$, and (b) $R=0.1 \mathrm{fm}$.
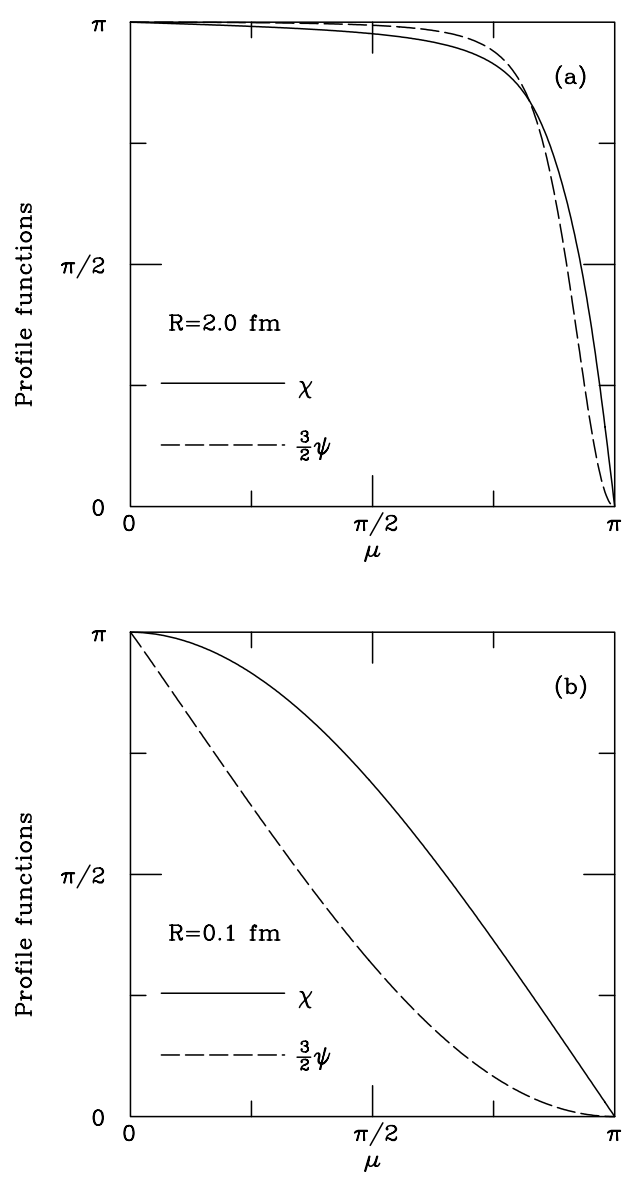

\section{References}

[1] T. Sakai, A. Buchmann, K. Yazaki and K. Shimizu, Nucl. Phys. A543 (1992) 661;

T. Sakai, K. Yazaki and K. Shimizu, Nucl. Phys. A594 (1995) 247 and references therein.

[2] R.L. Jaffe, Phys. Rev. Lett. 38 (1977) 195; 38 (1977) 617 (errata).

[3] E. Golowich and T. Sotirelis, Phys. Rev. D46 (1992) 354;

V.B. Kopeliovich, B. Schwesinger and B.E. Stern, Nucl. Phys. A549 (1992) 485;

G.L. Thomas, N.N. Scoccola and A. Wirzba, Nucl. Phys. A575 (1994) 623;

N. Kodama, M. Oka and T. Hatsuda, Nucl. Phys. A580 (1994) 445. 
Figure 2: The energy of the $\mathrm{SO}(3)$ Skyrmion on $S^{3}$ as a function of the hypersphere radius $R$ (solid line). The energy in the case of the "identity map", $\chi(\mu)=\pi-\mu$ and $\psi(\mu)=\frac{2}{3}(\pi-\mu)$, is also shown by the dashed line. The "identity map" is found to be a good approximation for small $R$.

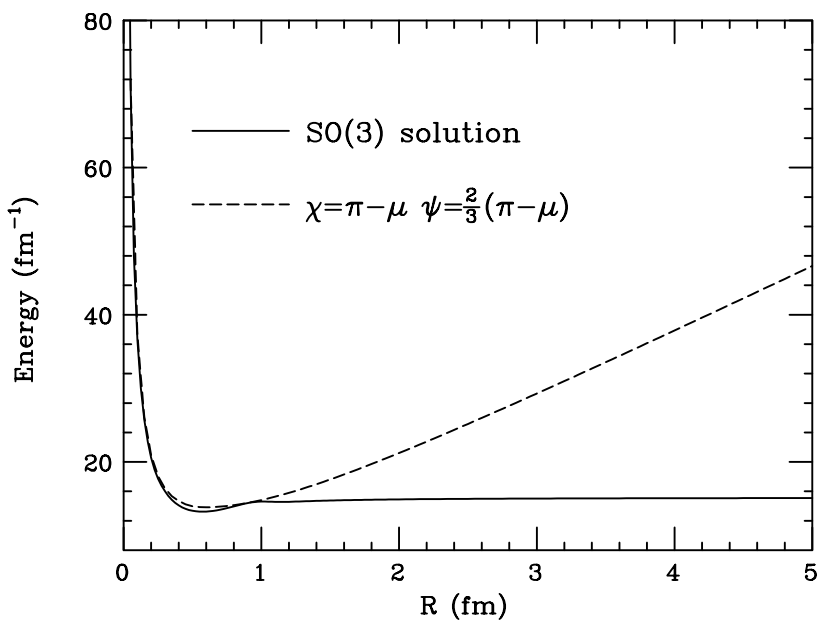

[4] A.P. Balachandran, A. Barducci, F. Lizzi, V.G.J. Rodgers and A. Stern, Phys. Rev. Lett. 52 (1984) 887;

A.P. Balachandran, F. Lizzi, V.G.J. Rodgers and A. Stern, Nucl. Phys. B256 (1985) 525.

[5] R.L. Jaffe and C.L. Korpa, Nucl. Phys. B258 (1985) 468;

S.A. Yost and C.R. Nappi, Phys. Rev. D32 (1985) 816.

[6] B.A. Shahbazian, A.O. Kechechyan, A.M. Tarasov and A.S. Martynov, Z. Phys. C39 (1988) 151 ;

B.A. Shahbazian, V.A. Sashin, A.O. Kechechyan and A.S. Martynov, Phys. Lett. B235 (1990) 208;

B.A. Shahbazian, T.A. Volokhovskaya, V.N. Yemelyanenko and A.S. Martynov, Nuovo Cimento 107A (1994) 2459;

A.N. Alekseev et al., Yad. Fiz. 52 (1990) 1612 [Sov. J. Nucl. Phys. 52 (1990) 1016].

[7] R. Longacre et al., Nucl. Phys. A590 (1995) 477c.

[8] S. Aoki et al., Phys. Rev. Lett. 65 (1990) 1729.

[9] R. Tamagaki, Prog. Theor. Phys. 85 (1991) 321;

M. Ishii, R. Tamagaki and A. Tohsaki, Prog. Theor. Phys. 92 (1994) 111. 
Figure 3: Baryon number densities $B^{0}(\mu)$ vs. the angle $\mu$ of the hypersphere for various hypersphere radii $R$.

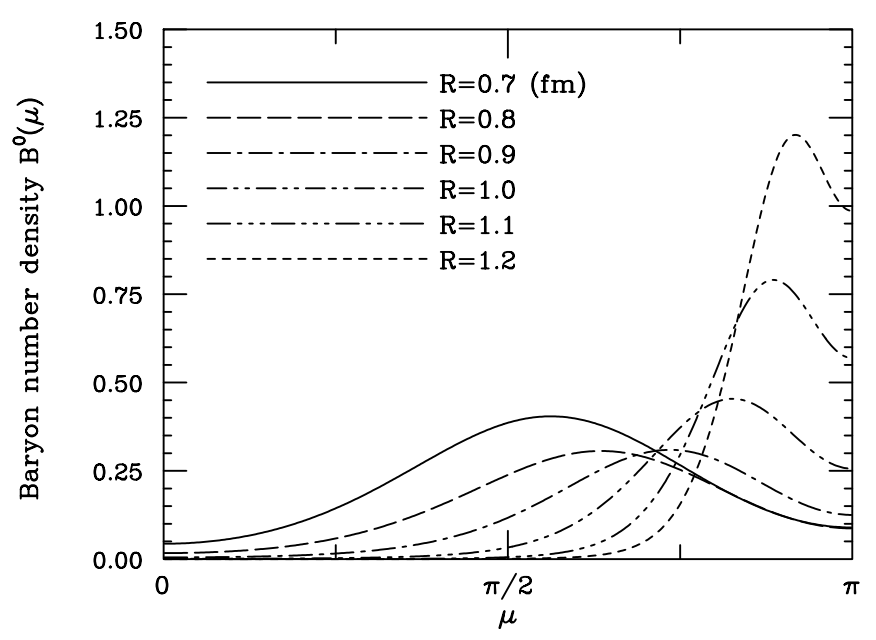

[10] H. Ichie, A. Hayashigaki, A. Suzuki and M. Kimura, Effective masses and sizes of N(939), $\Delta(1232)$ and $\mathrm{N}(1440)$ in nuclear medium, in Proc. Theoretical Physics Symposium in honor of Paulo Leal Ferreira (Sãn Paulo, August 1995), eds. V.C. Aguilera-Navarro, D. Galetti, B.M. Pimentel and L. Tomio (Editora Instituto de Física Teórica, São Paulo, 1995) p.247; M. Rho, Phys. Rev. Lett. 54 (1985) 767;

K. Saito and A.W. Thomas, Nucl. Phys. A574 (1994) 659.

[11] L. Castillejo, P.S.J. Jones, A.D. Jackson, J.J.M. Verbaarschot and A. Jackson, Nucl. Phys. A501 (1989) 801.

[12] N.S. Manton and P.J. Ruback, Phys. Lett. B181 (1986) 137;

N.S. Manton, Commun. Math. Phys. 111 (1987) 469.

[13] A.D. Jackson, A. Wirzba and L. Castillejo, Phys. Lett. B198 (1987) 315;

A.D. Jackson, A. Wirzba and L. Castillejo, Nucl. Phys. A486 (1988) 634.

[14] V.G. Makhankov, Y.P. Rybakov and V.I. Sanyuk, The Skyrme Model (Springer-Verlag, Berlin Heidelberg, 1993).

[15] I. Zahed and G.E. Brown, Phys. Rep. 142 (1986) 1.

[16] H. Forkel, A.D. Jackson, M. Rho, C. Weiss, A. Wirzba and H. Bang, Nucl. Phys. A504 (1989) 818. 
Figure 4: The change of the chiral order parameter $\sigma_{0}$ with respect to the hypersphere radius $R$.

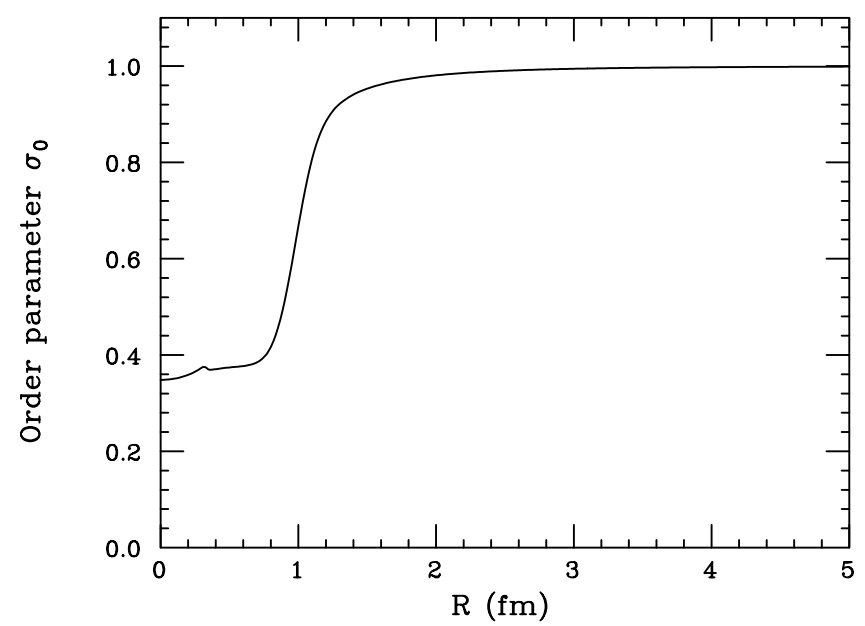

[17] A.I. Issinskiı̆, B.V. Kopeliovich and B.E. Shtern, Yad. Fiz. 48 (1988) 209 [Sov. J. Nucl. Phys. 48 (1988) 133]. 
Figure 5: The Skyrmion r.m.s. radius $\bar{r}$ defined by eq.(12) as a function of the hypersphere radius $R$ (solid line). The contributions from the upper half $(0 \leq \mu \leq \pi / 2)$ and the lower half $(\pi / 2 \leq \mu \leq \pi)$ of the hypersphere are also shown by dashed line and dot-dashed line, respectively.

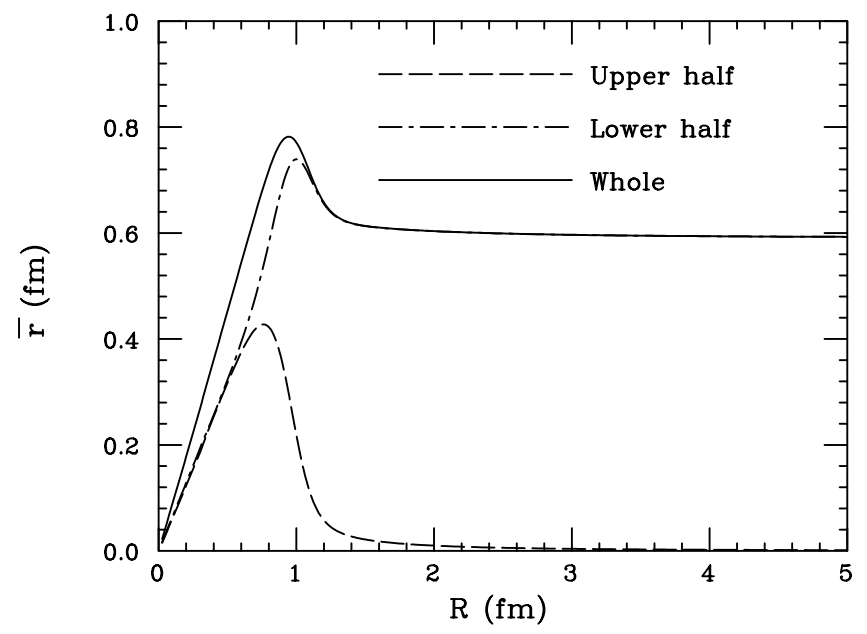

Figure 6: The comparison between the $\mathrm{SO}(3)$ and the $\mathrm{SO}(2) \times \mathrm{SO}(2)$ solutions on $S^{3}$. The energies, $E_{\mathrm{SO}(3)}$ and $E_{\mathrm{SO}(2) \times \mathrm{SO}(2)}$, are plotted as functions of the radius $R$.

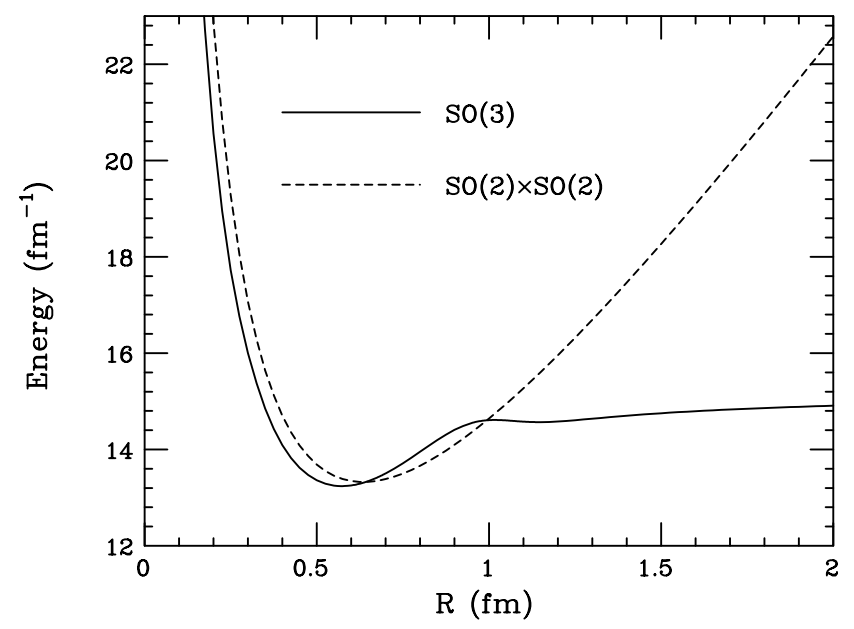

